\title{
Pour l'art et pour la technique. Former à la céramique (France, 1840-1940)
}

\section{Florent Le Bot}

\section{(2) OpenEdition}

Journals

Édition électronique

URL : https://journals.openedition.org/artefact/9224

DOI : $10.4000 /$ artefact.9224

ISSN : 2606-9245

Éditeur :

Association Artefact. Techniques histoire et sciences humaines, Presses universitaires du Midi

Édition imprimée

Date de publication : 11 septembre 2014

Pagination : 113-134

ISBN : 978-2-271-08150-6

ISSN : 2273-0753

Référence électronique

Florent Le Bot, «Pour l'art et pour la technique. Former à la céramique (France, 1840-1940) 》, Artefact

[En ligne], 2 | 2014, mis en ligne le 12 mai 2021, consulté le 24 août 2021. URL : http://

journals.openedition.org/artefact/9224 ; DOI : https://doi.org/10.4000/artefact.9224

\section{(c)}

Artefact, Techniques, histoire et sciences humaines est mise à disposition selon les termes de la Licence Creative Commons Attribution - Pas d'Utilisation Commerciale - Pas de Modification 4.0 International. 


\section{Pour l'art et pour la technique. Former à la céramique (France, 1840-1940)}

Florent $L E B O T^{1}$

\section{Résumé}

L'évolution de la formation aux métiers de la céramique, de 1840 à 1940, accompagne l'industrialisation et l'extension des débouchés, la nationalisation de l'enseignement technique, le développement de réseaux $d$ 'institutions par les acteurs publics et privés. Ces dynamiques sont observées depuis Limoges, avec son école de beaux-arts appliqués à l'industrie, depuis l'ENP de Vierzon et sa section céramique, et, enfin, à travers la création de l'Institut de la céramique française, en relation avec la manufacture nationale de céramique de Sèvres.

Mots-clés : art, céramique, enseignement, entreprise, porcelaine, technique.

\section{Abstract}

The evolution of training for ceramics trades from 1840 to 1940, went along with industrialization and expansion of markets, nationalization of technical education, the development by public and private actors of networks institutions. Limoges, with its fine arts school for industry, Vierzon, with its National professional school and its ceramic division, and finally, the creation of the Institute of French ceramics, in relation with the National manufacture of Sèvres ceramics, allow to analyze these dynamics.

Keywords : art, ceramic, China, education, firm, technical. 
Le thème de la formation aux métiers de la céramique s'inscrit dans les problématiques d'ensemble de la formation technique: entre les attentes, les réticences, les demandes et les offres des milieux industriels, les positions, parfois divergentes, des milieux éducatifs et les options et réalisations de l'État et des collectivités locales ${ }^{2}$, existe un certain nombre de contradictions et de tensions, parfois aussi de convergences. La contradiction se noue durablement entre besoins pratiques de l'industrie - y compris les besoins de centres industriels localisés spécifiques - et vision scolaire d'ensemble qui font de l'enseignement classique le point de référence dans les discours, pour s'en réclamer ou au contraire pour le déplorer. Dans le domaine de la céramique, c'est la référence à "l'art», sommet de la hiérarchie symbolique, qui alimente cette contradiction: forme-t-on des artistes ou des techniciens? Forme-t-on des décorateurs ou d'habiles ouvriers? Doit-on former des personnes se distinguant par leur goût ou par leur savoir-faire en matière technique? Le débat et les solutions retenues engagent l'État (à travers notamment les ministères du Commerce et de l'Instruction publique, à travers les directions des Beaux-Arts et de l'Enseignement technique), les collectivités locales (municipalités et conseils généraux), patrons et organisations patronales, associations professionnelles et d'anciens élèves. Ainsi, une histoire institutionnelle paraît nécessaire pour saisir le cadre de la transformation des métiers, des modes de production et des besoins de formation, sur toute la période de l'industrialisation depuis une protoindustrialisation associée à une offre de formation par des société savantes directement sortie de la Révolution et des Lumières, jusqu'au paysage du premier $\mathrm{xx}^{\mathrm{e}}$ siècle organisé par de nouveaux acteurs, institutions républicaines, syndicats patronaux et associations professionnelles, se rejoignant autour des besoins d'une industrie de masse mécanisée et dont les débouchés sont en expansion (de la porcelaine vaisselière et fantaisie jusqu'à la céramique technique)

S'agissant d'une action qui se joue à l'échelon national, mais également au niveau local, il faut souligner que, comme beaucoup de secteurs d'activités traditionnelles ${ }^{3}, l^{\prime}$ industrie céramique est dominée numériquement par des petites et moyennes entreprises regroupées pour la plupart dans des localités dédiées (ou quasiment $)^{4}$. Dans le domaine de la porcelaine vaisselière et fantaisie, Limoges domine avec ses trente-huit fabriques en 1907 (pic historique de la production de porcelaine, avec $330000 \mathrm{~m}^{3}$ de porcelaine cuite sortie des fours), environ 9000 ouvriers/ouvrières et peu de grandes entreprises (Charles Haviland, 2500 ouvriers; Théodore Haviland, 1000). Une production plus fruste sort des fabriques du Berry, à Vierzon (la principale manufacture, créée en 1816, y emploie environ 350 personnes en 1830; en 1903, Vierzon-ville compte sept fabriques de porcelaine dans lesquelles travaillent plus de 1500 personnes) $)^{5}$, ou à proximité (Foëcy, Mehun, Noirlac, etc.), ou encore à Châtres-sur-Cher, près de Blois. Il faut ajouter à ces territoires productifs, la manufacture nationale de céramique de Sèvres qui occupe une place de référence dans le secteur. Dans le domaine de la céramique technique, en expansion au $\mathrm{xx}^{\mathrm{e}}$ siècle, mentionnons 
Beauvais et l'Oise pour les produits de construction et les matériaux réfractaires (par exemple, «la manufacture de grès de Beauvais» dite manufacture Gréber, en service de 1868 à 1962, employant 35 personnes à la fin du $\mathrm{xIx}^{\mathrm{e}}$ siècle $)^{6}$ et Châlons-sur-Saône pour les céramiques sanitaires.

Ainsi, la spécialisation par type de produits s'établit sur une base géographique. Or, les questions d'enseignement technique, plus que tout autre domaine d'enseignement sans doute, impliquent une articulation, parfois conflictuelle, parfois fertile, entre volontés nationales, attentes locales, marges de négociation et décisions effectives. Ce sont ces «rapports délicats que l'on retrouve dans ces autres couples fondamentaux présidant à la conception du projet que sont les objec- tifs économiques et/ou idéologiques, le contenu professionnel et/ou général du savoir diffusé ${ }^{»}$. Les exemples localisés (Limoges, Vierzon, Beauvais) permettent d'éclairer une évolution dans la définition des besoins d'enseignement, de l'art vers la technique, en relation avec un processus d'industrialisation et de développement de la céramique industrielle. Ils signalent également une institutionnalisation croissante des rapports entre les milieux patronaux, les associations professionnelles et l'État, observable dans d'autres domaines, et dont la création de l'Institut de céramique française à Sèvres est l'expression la plus tangible. Il s'agit finalement d'examiner les transformations d'une filière de l'économie à travers les enjeux de formation.

\section{Entre art et industrie : un malentendu durable à Limoges}

\section{Former des décorateurs?}

La question de la formation de décorateurs pour les pièces de céramique à Limoges ne se pose vraiment qu'à partir des années 1840. Jusqu'à cette période, les porcelainiers font du blanc et ce n'est qu'occasionnellement, pour les pièces fantaisies (vases, statuettes, etc.), qu'un décor est ajouté; et encore, celui-ci est-il le plus souvent peint à Paris ou à Toulouse ${ }^{8}$. En 1844, David Haviland fonde à Limoges son propre atelier, créant les modèles dont il fournit les moules aux fabricants, avant de récupérer le produit qu'il met à décorer. Il embauche pour cela une centaine d'apprentis peintres encadrés par des maîtres, associant ainsi en un même lieu formation et production. S'ouvre alors une course à l'industrialisation qui conduit, en 1855, David et son fils aîné Charles à construire une manufacture réunissant pour la première fois fabrique et atelier de décoration. Au fil des ans, celle-ci ne va cesser de s'agrandir et de se mécaniser'. La réussite d'Haviland, particulièrement sur le marché nord-américain, contribue à convertir progressivement les autres fabriques à la décoration ${ }^{10}$, en même temps qu'à l'exportation: en 1900, ce sont les usines les plus importantes (18 sur 39) qui possèdent un atelier de décoration. Dans l'état actuel de notre documentation, nous ne savons pas si le modèle de formation retenu initialement par l'entreprise Haviland est repris 
par d'autres manufactures, ni même s'il perdure dans ladite manufacture. En tous cas, il ne passe pas inaperçu auprès de concurrents qui peuvent se sentir menacés: "Élèves et professeurs, dans les premiers temps, ne circulaient que par bandes, afin de pouvoir se défendre contre les menaces d'un mauvais coup ${ }^{11}$.»

Le processus d'industrialisation s'accompagne d'évolutions techniques en matière de décoration, ce qui n'est pas sans retentir sur les besoins de formation. Au cours des années 1860, la décoration par procédé d'impression se substitue progressivement à la décoration à la main. C'est d'abord la taille-douce (gravure sur cuivre) grâce à laquelle il est possible de reproduire en grand nombre des motifs monochromes sur papier, reportés par décalcomanie sur la porcelaine, qui sont ensuite enluminés au pinceau par des ouvrières. À partir des années 1880, la chromolithographie permet la reproduction en série de motifs en plusieurs couleurs sur les pièces. Les motifs sont imprimés, couleur par couleur, sur des feuilles de "chromo» enduites d'un vernis spécial. Ils sont reportés par décalcomanie sur l'objet en porcelaine par les ouvrières décalqueuses. La chromolithographie, mise au point en 1837 par Geoffroy Engelmann, puis adaptée à la porcelaine en 1847 par le parisien LouisAimé Césaire Macé, est utilisée notamment pour le célèbre service du président Hayes réalisé en 1880 par la manufacture Haviland $^{12}$. Son décor ne comporte que six couleurs quand, à la fin du siècle, les progrès techniques vont permettre $d^{\prime}$ obtenir des chromos de dix-huit couleurs.

Les nouvelles techniques d'impression induisent des bouleversements tant au niveau de la décoration que du point de vue des ouvriers. L'impression permet une standardisation des objets décorés. Elle accroît aussi considérablement la productivité des ateliers de décor: le travail long et minutieux du peintre en porcelaine est remplacé par celui plus simple d'une ouvrière décalqueuse. Il s'ensuit une diminution des prix de vente de la porcelaine de 20 à $40 \%$. En outre, la chromolithographie n'exige aucune qualification particulière. Cette déqualification du travail de décoration s'accompagne d'une féminisation de l'emploi: les ouvrières constituent jusqu'à la moitié, voire plus, de l'effectif des ateliers de décor. Les salaires sont faibles (une décalqueuse ne gagne que $30 \%$ du salaire d'un peintre, une enlumineuse $20 \%$ ) et les conditions de travail difficiles (insalubrité des ateliers). La spécialisation sur des parties de décor (fleuristes, sujettistes, fileurs, doreurs, etc.) devient la règle et les ouvrier(e)s acquièrent les savoir-faire strictement indispensables à l'accomplissement de leurs tâches: n'ayant «reçu qu'une éducation artistique très empirique et [étant] spécialisés dans tel ou tel genre, ils sont incapables le plus souvent de créer des modèles nouveaux. ${ }^{13}{ }^{»}$

Le procédé chromolithographique favorise l'intégration de l'atelier de décor à l'usine. Au début du $\mathrm{xx}^{\mathrm{e}}$ siècle, il demeure toutefois une quarantaine d'ateliers indépendants subsistant en marge des installations industrielles et intervenant sur des objets à décor riche ou décor spécifique impossible à réaliser en série et sur les rebuts des grandes fabriques. L'enjeu d'une formation de décorateur à la main hors de la fabrique concerne ainsi une main-d'œuvre qui va en se réduisant, qui travaille dans 
les fabriques n'employant pas (ou pas encore) les procédés d'impression ou dans des ateliers de décoration indépendants produisant des pièces uniques - «l'art décoratif» plutôt que «l'art industriel», pour reprendre une des distinctions de l'époque ${ }^{14}$.

Enfin, s'agissant de la création artistique proprement dite, les principales manufactures font appel à des artistes extérieurs. L'exemple le plus emblématique est celui de l'atelier d'Auteuil, ouvert en 1873 par Ch. Haviland et dirigé par le graveur Félix Bracquemond ${ }^{15}$. Toutefois, il existe également des décorateurs à domicile, appelés chambrelans, qui pourvoient les fabriques en modèles nouveaux.

\section{L'École municipale des beaux-arts appliqués à l'industrie}

Les modalités de l'industrialisation rencontrent les pratiques de la formation technique. En particulier, l'enseignement du dessin, dans une perspective de développement industriel et économique, apparaît comme élément constitutif de l'éducation technique au $\mathrm{xIX}^{\mathrm{e}}$ siècle ${ }^{16}$. Une société savante, la Société d'agriculture, des sciences et des arts, organise depuis 1804, à Limoges, un cours de dessin dans lequel se mêlent classiquement dessin linéaire et dessin décoratif ${ }^{17}$. À partir de 1824 , les cours s'y diversifient et, en 1846 , $\mathrm{s}^{\prime}$ ouvre un cours de modelage, puis en 1862 un autre de peinture sur porcelaine. L'offre ne semble pas correspondre aux attentes des fabricants et aux besoins de la production, puisque, dans les années 1860 , certains d'entre eux tentent d'organiser une école professionnelle ou, pour le moins, des cours publics pour les ouvriers. Toutefois, les moyens financiers leur font défaut.

En 1868, Adrien Dubouché (18181881) crée, à Limoges, l'École municipale des beaux-arts appliqués à l'industrie. Dubouché est particulièrement qualifié en terme de rapprochement arts et industries, puisqu'il est aussi bien collectionneur de céramique (en 1865, il prend la direction du musée de Limoges, auquel il offre sa collection; le musée prend son nom en 1876), lié aux amateurs d'art et au milieu artistique parisien (Charles Blanc, directeur de la Gazette des Beauxarts; Albert Jacquemart, historien de l'art et de la céramique, collectionneur; Auguste Louvrier de Lajolais, membre de l'Union centrale des beaux-arts appliqués à l'industrie; etc.), négociant en cognac et lié aux industriels de Limoges (en particulier à Henri Ardant et Charles Haviland).$^{18}$

L'objectif de l'École municipale des beaux-arts appliqués à l'industrie est à la fois de travailler à la renommée de la place, à la formation de spécialistes, peintres sur porcelaine et modeleurs, dont celle-ci a besoin, mais également de promouvoir "l'art appliqué à l'industrie", la production en série dans laquelle se lirait l'invention de l'artiste ${ }^{19}$ : «[...] former le goût du producteur est chose bien essentielle, mais ce n'est que la moitié de la tâche que nous devons accomplir: il est presque aussi nécessaire de former le goût de la foule qui consomme ${ }^{20} . »$ Le marchand Dubouché reformule ainsi un discours qui, depuis plusieurs décennies, exprime la nécessité d'éduquer les «classes montantes engagées dans l'imitation des consommations des classes les plus aisées ${ }^{21}{ }^{1}$. L'installation du musée au rez-de- 
chaussée du bâtiment qui accueille l'école contribue à alimenter l'inspiration des élèves dans la filiation de l'école de dessin de Mulhouse et de ses collections d'étoffes (1833), puis de son musée du Dessin industriel $(1857)^{22}$. L'enseignement de l'art par les modèles est un topos des milieux de l'enseignement artistique au XIX ${ }^{\mathrm{e}}$ siècle ${ }^{23}$.

L'école est une institution municipale, mais reçoit le soutien des fabricants (dont le principal, Charles Haviland, ami de Dubouché) sous forme de subventions annuelles. Ceux-ci apportent également leur concours à son fonctionnement et prêtent leurs fours. Mais, dès 1870, en plein marasme des affaires, ils retirent leurs subventions. Le lien n'est cependant pas rompu, notamment parce que le corps professoral demeure en partie constitué de spécialistes des manufactures, tel Charles Lissac, chef de l'atelier de décoration de Haviland et $\mathrm{C}^{\mathrm{ie}}$ et enseignant à l'école entre 1870 et 1874 .

L'école parvient, dans une certaine mesure, à adapter ses enseignements aux évolutions techniques et à l'industrialisation. Ainsi, en 1877, instaure-t-elle un cours de gravure qui offre l'occasion à Dubouché de proclamer: «Messieurs, on vient d'appliquer la vapeur à la décoration de la porcelaine, la science a trouvé l'impression et la science a bien fait. Elle permet de vulgariser des décors copiés sur les maîtres, ce qui naturellement va gêner les décors fabriqués trop vite par des artistes sans talent suffisant. [...] Nous ferons des décors d'un vrai mérite, d'un grand goût et d'un bon prix. [...] Vous trouverez peu ou pas d'ouvrage pour les peintures d'un talent vulgaire parce que l'impression fera mieux et meilleur marché que vous; en revanche, si vous le voulez, vous aurez un salaire très convenable pour les décors d'une véritable valeur ${ }^{24}$.» Par une inversion tout à fait intéressante, la valeur artistique résiderait désormais, selon Dubouché, dans la production en série, fruit du travail conjoint de l'art et de la science plutôt que dans l'objet unique. $C^{\prime}$ est prendre acte des progrès de l'industrialisation des décors, sans toutefois en mesurer toutes les implications en termes de nature et de besoin de formation.

\section{L’École nationale des arts décoratifs : une nationalisation pour l'art}

Malgré les adaptations, et au-delà de la conjoncture, l'École municipale des beaux-arts appliqués à l'industrie déçoit les fabricants qui n'y trouvent pas un personnel formé selon leurs convenances: «Mais là n'est pas le rôle [...] de Limoges qui n'est pas une école professionnelle; elle laisse l'apprentissage à l'atelier, se bornant à être une initiatrice d'art, à faire l'éducation artistique plutôt que technique de ses élèves ${ }^{25}$.» Qui former? Pour quelles tâches? Dans quelles conditions? Et pour quelles entreprises? Nos sources ne font pas la part entre les manufactures les plus importantes, organisant l'apprentissage en leur sein, mais également, du fait de l'industrialisation du décor, ayant recours à des ouvrières spécialisées réclamant peu de formation, et la myriade des petites et moyennes entreprises pour lesquelles la mécanisation est moins avancée et qui ne peuvent couvrir en interne leurs besoins en formation. C'est sans doute là le reflet d'une incertitude de l'époque 
à distinguer type de producteurs et de productions. La non-différenciation des catégories socio-économiques contribue à la confusion de l'offre de formation.

Le 15 juin 1881, la transformation de l'École municipale en École nationale des arts décoratifs (ENAD) sous les auspices de l'administration des Beaux-Arts (transformation qui participe d'un mouvement d'ensemble avec l'École nationale des arts industriels de Roubaix et l'École nationale des arts décoratifs $d^{\prime}$ Aubusson $^{26}$ ) ne modifie pas substantiellement les rapports avec les industriels d'une école qui n'est ni professionnelle, ni technique, ni d'apprentissage, mais consacrée à l'enseignement artistique, les Beaux-Arts refusant de s'impliquer dans la formation professionnelle et laissant aux initiatives locales, publiques et privées, le soin $d^{\prime}$ intervenir en la matière ${ }^{27}$. L'enseignement à l'ENAD comprend: le dessin linéaire et géométrique, la perspective et les éléments d'architecture; le dessin, le modelage et l'anatomie comparée; un cours de composition d'ornement; un cours d'histoire de l'art; la peinture de fleurs à l'eau, à la colle et à l'huile; des cours spéciaux pour différentes applications des arts du dessin à l'industrie (céramique, émail, gravure à l'eau-forte, etc.). En devenant nationale, l'École offre désormais un enseignement gratuit et des bourses, tandis que la formation était répartie sur deux niveaux, élémentaire et supérieur $^{28}$. À l'occasion de ce changement de statut, $l^{\prime}$ École se trouve rattachée, tout comme Aubusson, à l'École des arts décoratifs de Paris sous la direction commune de Louvrier de Lajolais (Dubouché étant d'ailleurs membre, depuis 1877, du Conseil supérieur de protection et de contrôle de l'école parisienne). «Ainsi, écrit Stéphane Laurent, se forma un trio original qui reposait sur un principe de réciprocité entre les enseignements devant améliorer la formation technique des élèves: l'École des arts décoratifs de Paris fournissait des modèles que les deux autres, qui disposaient d'ateliers de céramique et de tapisserie, étaient chargés d'exécuter ${ }^{29}$.»

À Limoges, des efforts sont entrepris pour répondre aux besoins de l'industrie locale, mais la dynamique de nationalisation de l'enseignement l'emporte qui accorde plus de place aux objectifs pédagogiques fixés à Paris qu'aux attentes locales. Par exemple, en 1891, a été créé à l'ENAD, à l'intention des chambrelans, un cours supérieur de composition appliquée à la céramique s'achevant par un concours entre auditeurs: "Les chambrelans ont déserté ce cours, dont l'enseignement, déclarent-ils, était trop au-dessus du niveau de leur instruction artistique, et s'adressait bien plutôt aux élèves femmes de l'école, qui là comme dans le cours de peinture céramique constituaient la grande majorité des auditeurs, bien qu'aucune d'elles ne se destine à la profession de décorateur sur porcelaines ${ }^{30}$.» Lors d'une enquête en 1896, sur la situation des industries d'art, des écoles et des musées d'art industriel en France, la chambre syndicale des fabricants de porcelaine de Limoges est interrogée quant à savoir si «l'industrie a [...] les artistes créateurs de modèles et les ouvriers d'art qui lui sont nécessaires, étant donnés les progrès nouveaux accomplis dans la fabrication?» Sa réponse est unanime: "Ce double personnel est devenu insuffisant à la fois comme nombre et comme valeur d'art, 
et il y a extrême urgence à se préoccuper de le fournir à l'industrie ${ }^{31}$.» Sans doute, là aussi, la notion d'art et les attentes des entrepreneurs en la matière auraient gagné à être définies précisément.

L'exemple de Limoges, dans la seconde moitié du XIX ${ }^{\mathrm{e}}$ siècle, illustre la difficulté à déterminer les besoins en termes de contenu d'enseignement en relation avec un secteur d'activité qui se transforme et $\mathrm{s}^{\prime}$ industrialise, et tandis que l'ensei- gnement tend à se définir autour d'enjeux nationaux. Soulignons également les contradictions, au niveau local, d'un patronat qui se plaint de ne pas trouver d'ouvrier formés et qui, dans le même temps, refuse dans une large mesure les formations institutionnalisées hors l'entreprise, sur le temps de travail ${ }^{32}$. La voix des patrons n'en est que plus inaudible dans les débats sur la formation.

\section{Pour l'art puis pour la technique : l'École nationale d'apprentissage de Vierzon}

\section{Les ambitions de l'ENP de Vierzon}

L'ENP de Vierzon a été étudiée par Michel Pigenet dans le cadre de sa thèse d'État pour l'avant $1914^{33}$, étude qui, dans notre perspective, nécessite d'être complétée pour la période postérieure. Dans un article consacré à l'enseignement technique à Vierzon, il signale le même type d'ambigüités et de difficultés que celles rencontrées dans le cas de l'École des arts décoratifs de Limoges ${ }^{34}:$ l'objectif annoncé $^{\prime}$ avec la mise en place de l'ENP est de préparer les enfants d'ouvriers à l'entrée dans l'industrie; du moins, c'est ainsi qu'une alliance de circonstances entre syndicats ouvriers, syndicats patronaux, milieux politiques de droite et de gauche le conçoivent. La mise en œuvre conduit progressivement à une formation de plus en plus élitiste qui détourne de cette école l'essentiel des enfants des milieux ouvriers.

La question d'une école spécifique fait notablement débat à partir de 1870 et l'un des responsables politiques local et national marquant pour cette réalisation, Henri Brisson, déclare en 1877 que «l'intégration dans l'enseignement "d'éléments raisonnés du commerce et de l'industrie" de même qu'une école professionnelle "serait admirablement placée à Vierzon" ${ }^{\prime 35}$ ». Il faut dire que l'agglomération de Vierzon, en pleine croissance démographique, compte environ 18000 habitants qui s'emploient pour partie dans la métallurgie, la verrerie ou dans la céramique, notamment «la Grande fabrique» qui compte environ 700 ouvriers à l'époque. Par ailleurs, Vierzon est avantageusement située sur les axes ferroviaires Paris-Toulouse et Nantes-Lyon et à proximité du canal du Berry. La formation des enfants passe alors par des formules d'apprentissage non codifiées. L'idée de créer une école manuelle d'apprentissage se veut à la fois une réponse à cette absence de cadre institutionnel, ainsi qu'une volonté de résoudre ce que l'on appelle durablement la «crise de l'apprentissage ${ }^{36} »$. Un 
projet de loi est examiné au Parlement dès 1880. Adopté en 1881, il débouche sur le décret du 9 juillet 1881 créant à Vierzon «une École nationale d'enseignement primaire supérieur et $\mathrm{d}^{\prime}$ enseignement professionnel préparatoire d'apprentissage destinée à servir de type pour les établissements de même nature qui seront fondés par application de la loi susvisée ${ }^{37}$ ». Cette école n'ouvre ses portes qu'en octobre 1887, $\mathrm{du}$ fait de problèmes de financement et de coordination entre pouvoirs publics locaux et nationaux. Il faut dire que l'ENP occupe un espace important et a nécessité l'extension de l'agglomération de Vierzon sur un quartier nouveau: elle occupe ainsi $40000 \mathrm{~m}^{2}$, pour former des élèves de 3 à 15 ans, c'est-à-dire de la maternelle à la troisième année de formation professionnelle, en passant par $1^{\prime}$ école primaire ${ }^{38}$. Le $1^{\text {er }}$ octobre 1887 , l'ENP proprement dite, c'est-à-dire la formation professionnelle, accueille 46 élèves.

\section{Une école qui s'éloigne de ses objectifs initiaux}

L'ENP de Vierzon va décevoir quant à la réalisation de certains objectifs pour lesquels il s'agissait, selon le projet de loi, «de développer chez les jeunes gens qui se destinent aux professions manuelles, la dextérité nécessaire et les connaissances techniques indispensables ${ }^{39}{ }^{\prime}$. Jules Ferry, lors de la pose de la première pierre, le 3 mai 1883, précise: "Nous ne voulons pas créer à Vierzon une école professionnelle qui double et qui copie les écoles d'art et métiers de Chalons, $d^{\prime}$ Aix et d'Angers. Non ces écoles ont un but déterminé: elles se proposent de former des contremaîtres, des sous-officiers pour l'armée du travail; ici nous voulons préparer des soldats pour cette armée... ${ }^{40} »$

Le parcours de formation se partage entre enseignement général et professionnel. Le premier correspond au programme exigeant des écoles primaires supérieures: français, mathématiques, histoire-géographie, langues vivantes, physique, chimie, histoire naturelle, morale, chant et gymnastique. Le second, à travers des heures d'atelier organisées autour de six sections, correspond aux activités manufacturières locales: fer, bois, dessin industriel et d'ornement, manipulations chimiques et porcelaine.

Si l'on se réfère au nombre d'inscrits, l'ENP est un succès: 46 en 1887, 246 en 1894, 300 en 1900, 355 en 1906. Mais, s'agissant des objectifs initiaux - à grands traits, former des jeunes de la région pour les industries locales -, le contrat n'est pas tout à fait rempli, d'une part, parce que, les coûts d'équipement et d'entretien des élèves excédant les possibilités des classes populaires, le recrutement se fait de plus en plus hors de la région, d'autre part, parce que les objectifs pédagogiques sont très ambitieux. En 1895, l'ENP de Vierzon (comme les autres ENP d'ailleurs) devient un centre de préparation aux Arts et métiers (Arts et métiers qui délivrent le titre d'ingénieur à partir de 1907) plutôt qu'à l'emploi de contremaître. Les élèves qui auraient pu constituer la «clientèle naturelle» de l'école et des usines locales ne dépassent pas le stade d'une présélection sur dossier qui s'instaure alors de manière plus ou moins assumée. 


\section{La section de céramique : de l'art à la technique}

L'exemple de la céramique illustre les difficultés rencontrées pour mettre en place une formation adaptée aux besoins locaux en matière de main-d'œuvre qualifiée.

Inscrite dès l'origine au programme de l'École, la préparation aux métiers de la porcelaine délaisse très vite les spécialités les plus répandues de la branche, notamment celle du façonnage pour lesquelles, affirme le directeur de l'École, «un séjour de quelques mois dans une usine [en apprend] plus que tous les cours du monde ${ }^{41} »$. Échappent à cet abandon les formations censées permettre aux élèves «de devenir autre chose que des manœuvres sans aucune chance d'avenir ", soit de l'avis du directeur, ceux qui «appliquent le dessin»: peinture, modelage et sculpture. Or, l'industrie porcelainière est en pleine mécanisation et ces tâches sont remplies par des machines plutôt que par des artistes ou même d'habiles ouvriers décorateurs. Du coup, les élèves se raréfient et, en 1895, le ministère décide la suppression de la section. Dans un rapport au conseil d'administration du 18 juillet 1896, le directeur de l'ENP reconnaît que les usines de Vierzon ne réclament ni peintres, ni décorateurs: «Partout où je suis allé [dit-il], j'ai toujours vu que les fabricants se souciaient par-dessus tout de produire beaucoup et à bon marché. Le côté artistique est celui qui les intéresse le moins ${ }^{42}$.» Ce constat renvoie à une division géographique du travail qui conduit Limoges à occuper le créneau de la qualité, voire du luxe, concurrençant parfois la manufacture de Sèvres pour des commandes prestigieuses, tandis que, dans le reste de la France, et d'abord à Vierzon et dans le Berry, la production destinée à un marché plus large s'avère plus frustre. Ainsi, la main-d'œuvre nécessaire à l'industrie de Vierzon et de sa région n'a besoin d'être formée qu'à travers un apprentissage non codifié dans les usines, voire dans des cours du soir organisés par la municipalité.

Il faut attendre l'année 1920 pour qu'une section Céramique réapparaisse à l'ENP, encore qu'il ne s'agisse plus de former des peintres, modeleurs ou sculpteurs, mais des techniciens pour la céramique industrielle (vaisselle, carreaux, émaux, porcelaines électrotechniques, tuiles, briques, tuyaux, poterie, céramique sanitaire, produits réfractaires, industries du silicate et du verre, etc.). L'initiative en revient à un industriel porcelainier de Vierzon, Marc Larchevêque, qui a enseigné dans l'ancienne section de céramique et qui, à partir de 1911, plaide au sein du conseil d'administration pour cette renaissance ${ }^{43}$. Le projet reçoit le soutien en 1913 de la direction de l'Enseignement technique (au sein du ministère du Commerce jusqu'en 1920), mais la guerre éclatant, les travaux sont remis à plus tard et la section n'ouvre finalement qu'en 1920. Il s'agit d'une section de formation à la céramique industrielle et aux produits vitrifiés (porcelaine et grès) qui débouche sur un diplôme de fin d'études d'élève breveté de l'ENP de Vierzon de céramique industrielle et produits vitrifiés, devant permettre aux élèves qui en étaient pourvus, après deux années normales à l'ENP, puis deux années spéciales, de devenir cadres techniciens dans les usines ou d'être admis sans concours à l'École nationale supérieure de céra- 
mique de Sèvres ${ }^{44}$. Marc Larchevêque fut lui-même enseignant dans cette section jusqu'en 1931, assisté d'anciens élèves de l'école de Sèvres. Par ailleurs, il aménage au sein de l'ENP, un laboratoire d'essais mécaniques (chimie, modes de cuisson, résistance) dont les travaux bénéficient à l'ensemble de la profession ${ }^{45}$. La classe est désormais accolée au laboratoire, plutôt qu'au musée et à ses collections comme à Limoges. Cette association formation-recherche, classe-atelier-laboratoire, qui se généralise dans beaucoup de branches et de disciplines durant les années 1920, paraît l'indice d'une évolution du regard porté sur la pratique professionnelle, sur le processus de production et sur le produit lui-même, prolongeant, accompagnant et, sans doute aussi, encourageant les transformations de cette branche dans le sens de l'industrialisation et aussi du déploiement vers les céramiques techniques. La création de $l^{\prime}$ Institut de la céramique française $\mathrm{s}^{\prime}$ inscrit pleinement dans ce processus.

\section{L'Institut de la céramique française : une volonté de mise en ordre de l'enseignement technique céramique}

\section{L'Union céramique et chaufournière et la direction de l'Enseignement technique à l'initiative}

Les années 1920 semblent propices à ce que se dégage et s'organise un ordre $\mathrm{du}$ technique, en parallèle à l'ordre de l'enseignement secondaire et plus ou moins dans la continuité de l'enseignement primaire, pour une partie de son public $^{46}$. La création, en 1912, d'une École normale (pas encore supérieure) de l'enseignement technique (ENET), appelée à former les maîtres des ENP (type Vierzon) et des écoles pratiques commerciales et industrielles (EPCI, type Beauvais infra ${ }^{47}$, anticipait cette mise en ordre (le primaire ayant Saint-Cloud et Fontenay, le secondaire Ulm et Sèvres) réalisée par la loi d'Astier-Cuminal de 1919, relative à l'organisation de l'enseignement technique industriel et commercial.

Dans la céramique, cette mise en ordre passe par la création d'un Institut de la céramique française (ICF), une structure initiée par un syndicat patronal du secteur, l'Union céramique et chaufournière de France (UCCF), avec le soutien de $l^{\prime}$ État ${ }^{48}$. L'idée est en discussion dès avant la Grande guerre. Ainsi, lors du comité directeur de l'UCCF du 3 décembre 1913, la mise en place de ce «qu'on pourrait appeler Institut de mécanique appliquée et de céramique technique» est envisagé sur le modèle allemand, pays dans lequel des "écoles céramiques aident les fabri-

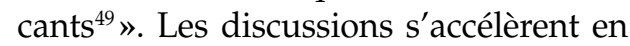
1917. M. Despret, chef du service technique de la céramique et de la verrerie au ministère du Commerce, promeut le projet devant plusieurs instances syndicales professionnelles ${ }^{50}$. Ces échanges 
entre administrations et représentants patronaux sont d'ailleurs à replacer dans un contexte au cours duquel le ministre du Commerce, Étienne Clémentel, mène une politique visant à renforcer la coordination entre l'État et l'industrie par l'institution d'interlocuteurs représentatifs (création de la Confédération générale de la production française - CGPF, en 1919, de la Confédération générale de l'artisanat français - CGAF, en 1922). L'Union céramique et chaufournière, devenue en 1917 le Syndicat des fabricants de céramique de France, avant de se transformer en Confédération des industries céramiques de France, groupant, à partir de 1937, l'essentiel des fédérations et syndicats nationaux professionnels céramiques, participe de l'institutionnalisation et du renforcement de la représentation patronale durant l'entre-deux-guerres ${ }^{51}$.

L'ICF est finalement créé à l'automne 1917 par le Syndicat des fabricants de produits céramiques de France ${ }^{52}$, reconnu d'utilité publique par la loi du 10 août $1920^{53}$ et installé en mars 1922. Ses statuts le définissent en tant $q u^{\prime}$ «établissement d'enseignement supérieur libre», ayant «pour but de constituer ou d'élargir les cadres $\mathrm{du}$ personnel instruit d'ingénieurs, de contremaîtres ou d'ouvriers dont l'industrie céramique française a besoin et d'accroître les moyens techniques et artistiques propres à favoriser son développement et son extension à l'étranger ${ }^{54}$ ». L'ICF «se propose également d'entreprendre ou d'encourager des publications ou des bibliographies relatives à la céramique ${ }^{55} »$. Ses principaux moyens d'action sont «une école supérieure de céramique», ainsi $\mathrm{qu}^{\prime}$ «un laboratoire de recherche d'essais et d'en- seignement ${ }^{56} »$. L'Institut participe à la réorganisation de l'existant et à la création de nouvelles écoles ou de nouveaux cours dédiés à la céramique (Fig. 1). Il s'agit, selon les termes de l'un des responsables de l'Association amicale des anciens élèves de l'École nationale supérieure de céramique de Sèvres, associée au projet, "de centraliser et d'organiser l'enseignement de la céramique à tous les degrés ${ }^{57}$ ». Au sein de l'ICF, «un Comité d'enseignement et de recherches techniques surveille et dirige en étroite collaboration avec le conseil [d'administration] les progrès des élèves et des recherches dans les différentes écoles ou laboratoires rattachés à $1^{\prime}$ Institut ${ }^{58}{ }^{\prime}$.

\section{L'ICF, Vierzon, Beauvais et l'Association d'enseignement technique céramique}

Ainsi, l'ICF contribue par un soutien financier à la renaissance de la section céramique de l'ENP de Vierzon avec pour objectif la formation de contremaîtres et de techniciens pour les produits vitrifiés.

Il participe également à la création d'une section Céramiqueà l'école pratique de commerce et d'industrie (EPCI) de Beauvais qui doit former des ouvriers qualifiés pour les produits céramiques du bâtiment. L'ouverture d'un cours pour la céramique technique à Beauvais remonte au $1^{\text {er }}$ octobre 1924. Dès 1930, il est prévu de le transformer en section grâce à une subvention de 600000 francs, la moitié prise en charge par l'État, $1 / 6^{\mathrm{e}}$ par la ville de Beauvais sous forme d'un terrain, $1 / 6^{\mathrm{e}}$ par le département de $1^{\prime}$ Oise et $1 / 6^{\mathrm{e}}$ par $1^{\prime} \mathrm{ICF}^{59}$. Son ouverture est effective au $1^{\mathrm{er}}$ octobre 1935: 
«Le but de cette section est la formation d'ouvriers de qualité destinés principalement aux industries de la céramique $\mathrm{du}$ bâtiment, étant entendu que ces ouvriers auront reçu à l'École une formation permettant de faire d'eux, lorsqu'ils auront acquis en usine l'expérience et la maturité nécessaires, des chefs d'équipes, contremaîtres ou employés techniques. La durée des études est de trois années pendant lesquelles les travaux d'atelier et l'enseignement pratique occupent une place prépondérante. Une partie de l'enseignement général complète l'instruction primaire des élèves ${ }^{60}$. »

En 1935, des projets similaires existent pour Limoges, Châlons-sur-Saône, etc.

Par ailleurs, une Association de l'enseignement technique céramique est reconnue d'utilité publique par décret du 2 janvier 1930. Son président est également vice-président de $\mathrm{l}^{\prime} \mathrm{ICF}^{61}$. Cette association est présentée comme «l'un des moyens d'exécution tant de la Confédération des industries céramiques de France que de l'Institut de céramique française». Elle a entre autres comme objet «la réalisation de cours pratiques et théoriques ayant trait à l'industrie céramique, l'organisation de congrès scientifiques et techniques ${ }^{62}$. Elle est en outre chargée, sous l'égide de l'Institut de céramique française, de contribuer à l'organisation et au contrôle de certains services d'études et de recherches susceptibles de répondre aux desiderata des divers syndicats professionnels céramiques constituant la Confédération ${ }^{63}$.» En matière de formation, il s'agit pour l'essentiel de proposer des cours de perfectionnement aux ouvriers qualifiés et aux contre- maîtres à Paris et dans les localités de l'industrie céramique.

L'ensemble du dispositif de formation aux métiers de la céramique, tel qu'il est présenté à travers la figure 1, correspond à la volonté des dirigeants des syndicats professionnels d'adapter les formations aux évolutions du secteur et aux besoins de leurs activités ${ }^{64}$. Cette volonté rencontre celle des pouvoirs publics, en particulier celle de la direction de l'Enseignement technique, d'organiser l'enseignement technique en général, l'enseignement technique céramique en particulier, de manière rationnelle et ordonnée, tel que l'indique Hippolyte Luc, son directeur de 1934 à 1944, dans un article de $1938^{65}$. Il reste à vérifier à partir de sources à repérer la réalité de la «tutelle», selon le mot de Luc, du Comité d'enseignement de la céramique française sur le dispositif de formation, le fonctionnement et les résultats effectifs de cet ensemble présenté en ordre: flux d'élèves et répartitions de ceux-ci en fonction des écoles, contenus des formations, compétences acquises par les élèves, emplois obtenus par ceux-ci à la sortie, etc. Les difficultés de l'ICF à collecter des souscriptions auprès des industriels, puis la taxe d'apprentissage (même si une part de ses ressources, que nous n'avons pu quantifier en l'état de la recherche, provient des subventions publiques), sont sans doute l'indice d'une marge entre planification envisagée et réalisations effectives ${ }^{66}$. Il $\mathrm{n}^{\prime}$ en demeure pas moins qu'une prise en compte des besoins de formation en relation avec les évolutions du secteur est à l'œuvre, tant du côté patronal que du côté des pouvoirs publics, sous les encouragements des 

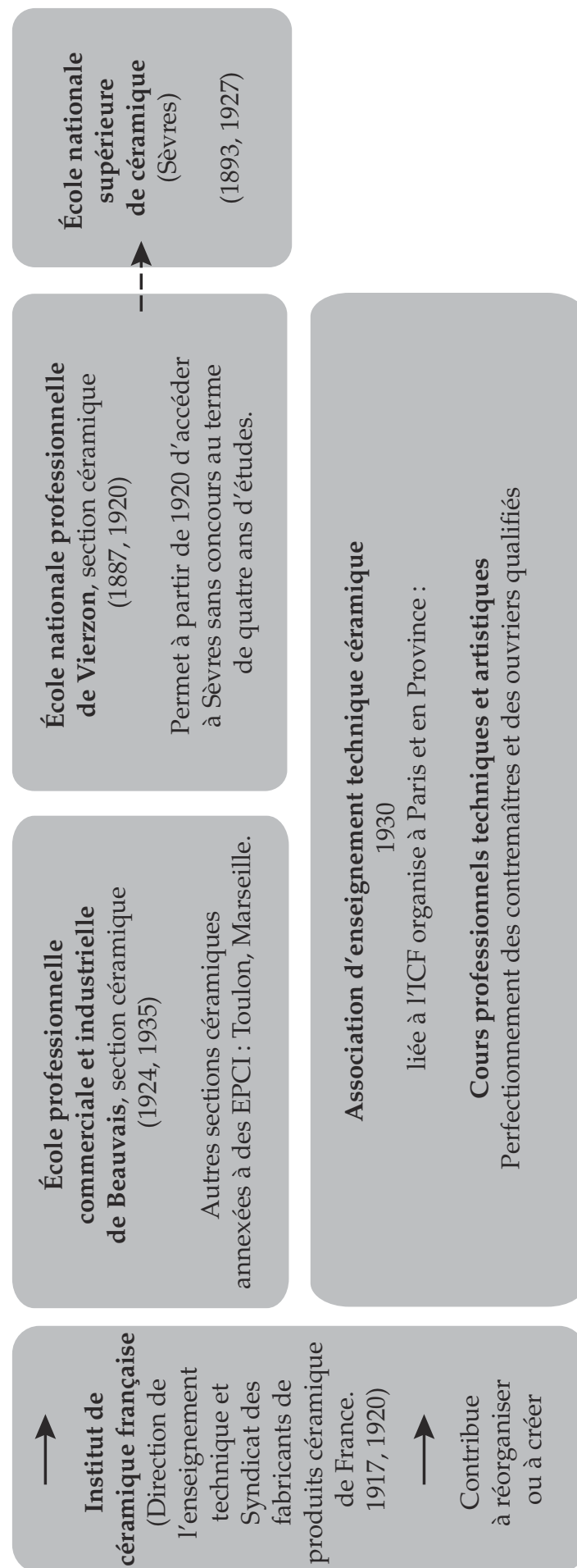

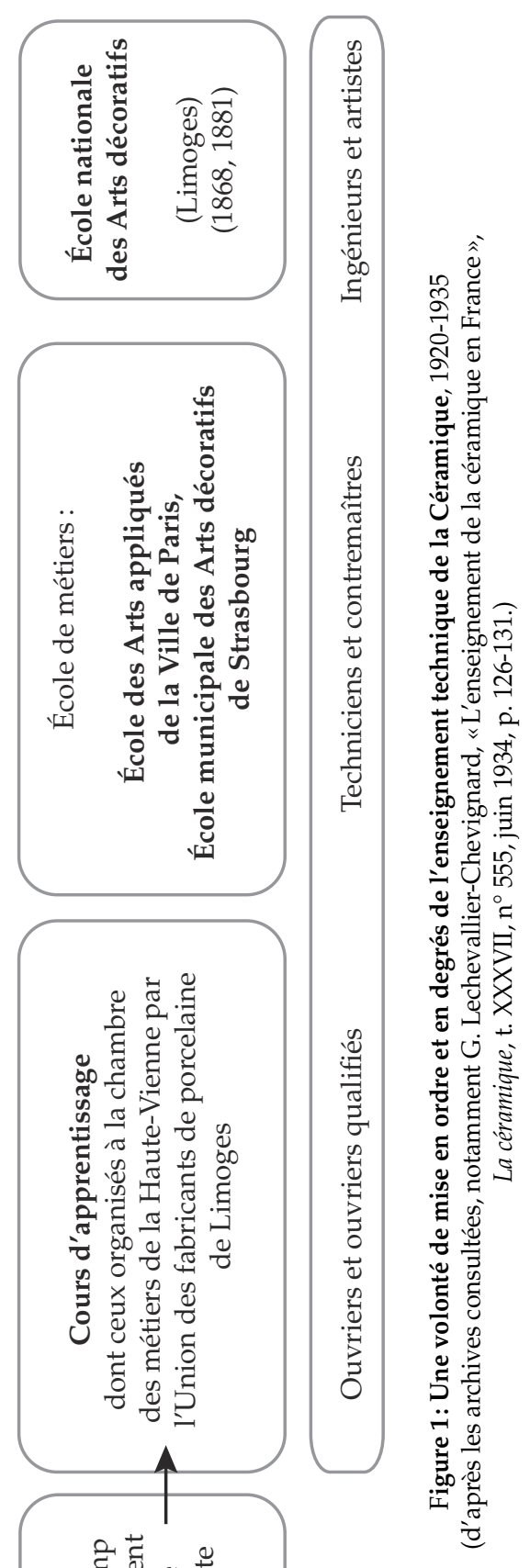

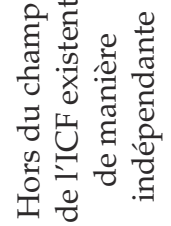


milieux enseignants et de la recherche, des associations d'anciens élèves et de professionnels. La réorganisation de l'École nationale de céramique de Sèvres, à laquelle contribue l'ICF, participe de cette prise en compte.

\section{L'ICF et l'École nationale supérieure de céramique de Sèvres}

Dans le paysage de la céramique française, Sèvres occupe une position particulière, gardant la main sur des savoir-faire de haute technicité à l'ancienne pour une production luxueuse de prestige, nécessitant une main-d'œuvre compétente. Dès 1879, la formation des artistes, des artisans et du personnel de la manufacture nationale est assurée par l'École d'apprentissage de la manufacture, puis École spéciale d'apprentissage. L'initiative en revient à la Commission de perfectionnement de la manufacture présidée par Charles Blanc, à laquelle participe Dubouché, Louvrier de Lajolais, Jacquemart, etc ${ }^{67}$. L'administration des Beaux-Arts considère alors l'enseignement comme un instrument de revalorisation de la production des manufactures nationales ${ }^{68}$.

La création, en 1893, de l'École de céramique annexée à la manufacture de Sèvres répond à une autre ambition. Accessible par voie de concours, celle-ci est destinée à la formation de collaborateurs pour l'industrie privée. L'enseignement d'abord durant cinq, puis quatre années, se divise alors entre une section artistique et une section technique. Dans les années 1900-1910, les industriels, à travers leurs syndicats professionnels et par le biais de l'Association amicale des anciens élèves de Sèvres, plaident pour que cette séparation soit abandonnée et que l'enseignement s'oriente vers la seule préparation des techniciens. En 1911, le programme des études est entièrement révisé. L'enjeu de la réorganisation réside dans la part respective de l'artistique et du technique dans l'enseignement. Le temps consacré à la technologie céramique, aux travaux de laboratoire, aux manipulations de chimie, etc., est augmenté de manière importante ${ }^{69}$. Par un arrêté du 13 mai 1919, l'institution prend la dénomination d'École nationale de céramique de Sèvres.

Le $1^{\text {er }}$ octobre 1926, la tutelle de l'École est confiée à la direction générale de $l^{\prime}$ Enseignement technique, tandis que la manufacture demeure administrée par celle des Beaux-Arts. La séparation entre les deux entités (qui conserve une même direction jusqu'en 1938) est actée à la suite d'un vote du parlement en décembre 1930. En 1927, une nouvelle définition des missions de l'École s'accompagne d'un changement de nom, qui devient l'École nationale supérieure de céramique.

Dès 1920, l'École est passée sous l'influence de l'ICF. Ainsi, l'Institut est représenté au conseil d'administration de la manufacture, puis, après la séparation de 1930, de l'École pour laquelle elle verse des subventions. Celle-ci accueille, par convention, les locaux de l'ICF et notamment son laboratoire de recherche qui, en retour, doit mettre à la disposition de la manufacture le fruit de ses travaux. Le personnel de $l^{\prime}$ Institut intègre le corps enseignant de la manufacture. Cette collaboration s'accompagne d'une nouvelle modification de l'enseignement à Sèvres, un 
large remaniement des programmes de l'École pour tenir compte de l'assimila- tion désormais complète de celle-ci aux écoles des arts et métiers (Fig. 2).

\begin{tabular}{|c|c|c|c|}
\hline \multicolumn{4}{|c|}{ Répartition hebdomadaire des matières enseignées } \\
\hline \multirow{2}{*}{ Cours } & $1^{\text {re }}$ année & $2^{\mathrm{e}}$ année & $3^{\mathrm{e}}$ année \\
\hline & heures & heures & heures \\
\hline Chimie & 1,5 & 1,5 & - \\
\hline Technologie & 1,5 & 1,5 & 1,5 \\
\hline Mathématiques & 4,5 & - & - \\
\hline Mécanique-électricité & - & 4 & - \\
\hline Histoire des styles & $1,5 / 2(?)$ & $1,5 / 2(?)$ & - \\
\hline Composition décorative & - & $1,5 / 2(?)$ & - \\
\hline Histoire de la céramique & - & - & 1 \\
\hline Hygiène et législation ouvrière & - & - & 1 \\
\hline Comptabilité industrielle & - & - & 1 \\
\hline Géologie minéralogie & 1 & - & - \\
\hline Constructions civiles & - & 1,5 & 1,5 \\
\hline Culture littéraire & 1 & 1 & 1 \\
\hline \multicolumn{4}{|c|}{ Exercices pratiques et manipulations } \\
\hline Technologie appliquée & 3 & 3 & 8 \\
\hline Mathématiques & 1 & 1 & - \\
\hline Mécanique-électricité & - & - & 4 \\
\hline \multicolumn{4}{|c|}{ Travaux pratiques } \\
\hline Laboratoire chimie & 6 & 6 & - \\
\hline Laboratoire céramique & 6 & 8 & 15 \\
\hline Atelier de fabrication & 4 & 7 & 7 \\
\hline Modelage & 3,5 & - & - \\
\hline Décoration céramique & 1,5 & - & - \\
\hline \multicolumn{4}{|c|}{ Dessin } \\
\hline Dessin industriel & 5 & 5 & 5 \\
\hline Dessin à vue & 3 & 3 & - \\
\hline Études & 12 & 12 & 10 \\
\hline Total & 56 & 56 & 56 \\
\hline
\end{tabular}

Figure 2: Une École pour l'industrie

(Bulletin de l'Association des anciens élèves, 1934, n²2, p. 37).

Cette réorganisation eut pour premier impératif l'identification des connaissances exigées des candidats aux concours d'admission et, en second lieu, l'utilisation rationnelle des ateliers et du matériel important dont l'École disposait à la suite d'un aménagement financé en partie par l'ICF.
«Le problème qui se posait, était de donner aux jeunes gens, en trois années d'études, et compte tenu du niveau des connaissances de base dont ils ont à faire preuve pour l'admission, la formation technique complète que doit posséder un ingénieur céramiste, accompagnée des éléments de culture générale 
indispensables à un futur chef et encore $\mathrm{du}$ minimum d'éducation du goût dont ne peut se passer un technicien céramiste dans certaines branches de son activité. L'ensemble du résultat cherché a été obtenu par un dosage judicieux des cours théoriques, d'exercices d'application, de manipulations et de travaux pratiques entre lesquels on s'est efforcé d'établir un synchronisme profitable $^{70}$.»
En 1934, seuls un peu plus de $6 \%$ des anciens élèves se rangent dans la catégorie «artisans céramistes d'art» contre $15 \%$ de décorateurs en 1910. En revanche, $55 \%$ se classent parmi les dirigeants et les ingénieurs de l'industrie céramique (Fig. 3). Par ailleurs, la figure 4, correspondant à des chiffres plus tardifs (1943), permet de préciser la diversité des débouchés de l'École (nous ne savons toutefois pas sur quelles données brutes ces statistiques s'appuient).

\begin{tabular}{|l|c|}
\hline \multicolumn{1}{|c|}{ Statistique des professions exercées par les anciens élèves de Sèvres } & en \% \\
\hline $\begin{array}{l}\text { Directeurs-généraux, directeurs d'usines, sous-directeurs, directeurs techniques } \\
\text { d'usines céramiques }\end{array}$ & 20,8 \\
\hline Ingénieurs, chefs de services, chef de fabrication, chimistes & 32,8 \\
\hline Ingénieurs-représentants & 4 \\
\hline Exerçants dans l'enseignement technique & 4,8 \\
\hline Artisans céramistes d'art & 6,4 \\
\hline chefs d'industries diverses matières (grès, etc.) & 3,2 \\
\hline Chefs d'industrie ou administrateurs-délégués d'entreprises hors céramiques & 11,2 \\
\hline Sans profession ou professions diverses en dehors de la céramique & 16,8 \\
\hline
\end{tabular}

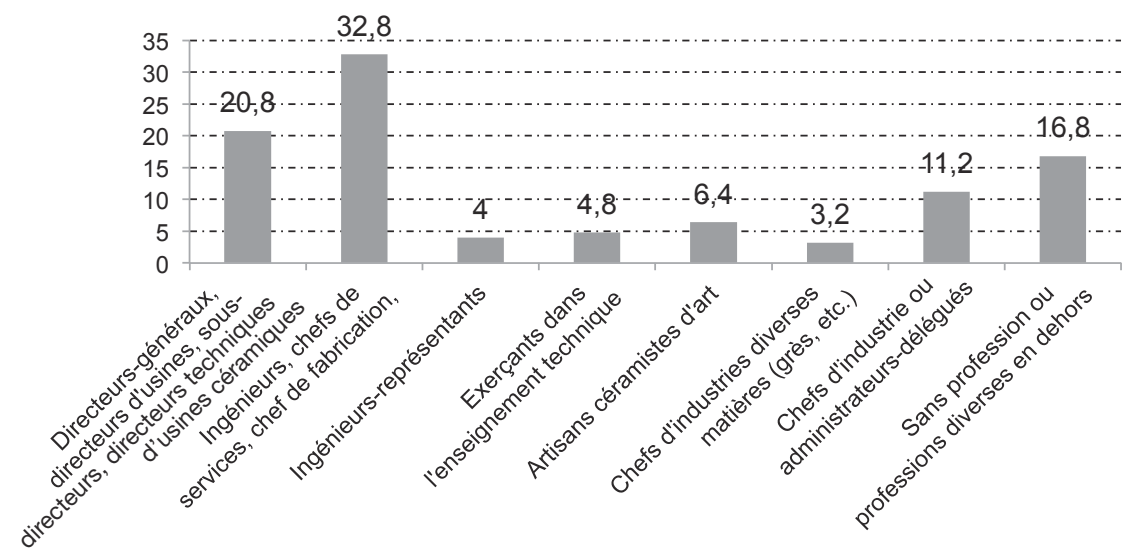

Source: Association amicale des anciens élèves de l'Ecole nationale supérieure de céramique de Sèvres. Bulletin annuel, $n^{\circ} 22,1934, p .28$

Figure 3: Statistiques des professions exercées par les anciens élèves de Sèvres, 1934, en \% (Association amicale des anciens élèves de l'École nationale supérieure de céramique de Sèvres, Bulletin annuel, $\mathrm{n}^{\circ} 22,1934$, p. 28). 


\begin{tabular}{|l|c|}
\hline \multicolumn{1}{|c|}{ Répartition industrielle des anciens élèves. } & en \% \\
\hline briques, tuiles, terres cuites de construction & 7,46 \\
\hline carreaux de grès et dallages & 6,71 \\
\hline céramique et verrerie d'art et de fantaisie & 11,93 \\
\hline chromolithographie céramique et industrielle & 0,75 \\
\hline couleurs et émaux céramiques & 1,49 \\
\hline crayons & 0,75 \\
\hline décoration céramique & 0,75 \\
\hline émaillerie & 2,98 \\
\hline enseignement technique & 1,47 \\
\hline faïencerie & 13,42 \\
\hline grès (poterie et tuyaux) & 2,98 \\
\hline ingénieurs-conseils pour la céramique & 2,98 \\
\hline porcelainerie & 7,48 \\
\hline produits céramiques sanitaires & 5,22 \\
\hline produits céramiques réfractaires & 9,7 \\
\hline représentation commerciale ou industrielle & 3,73 \\
\hline services et laboratoires d'études & 1,49 \\
\hline professions diverses autres que céramiques & 12,68 \\
\hline
\end{tabular}

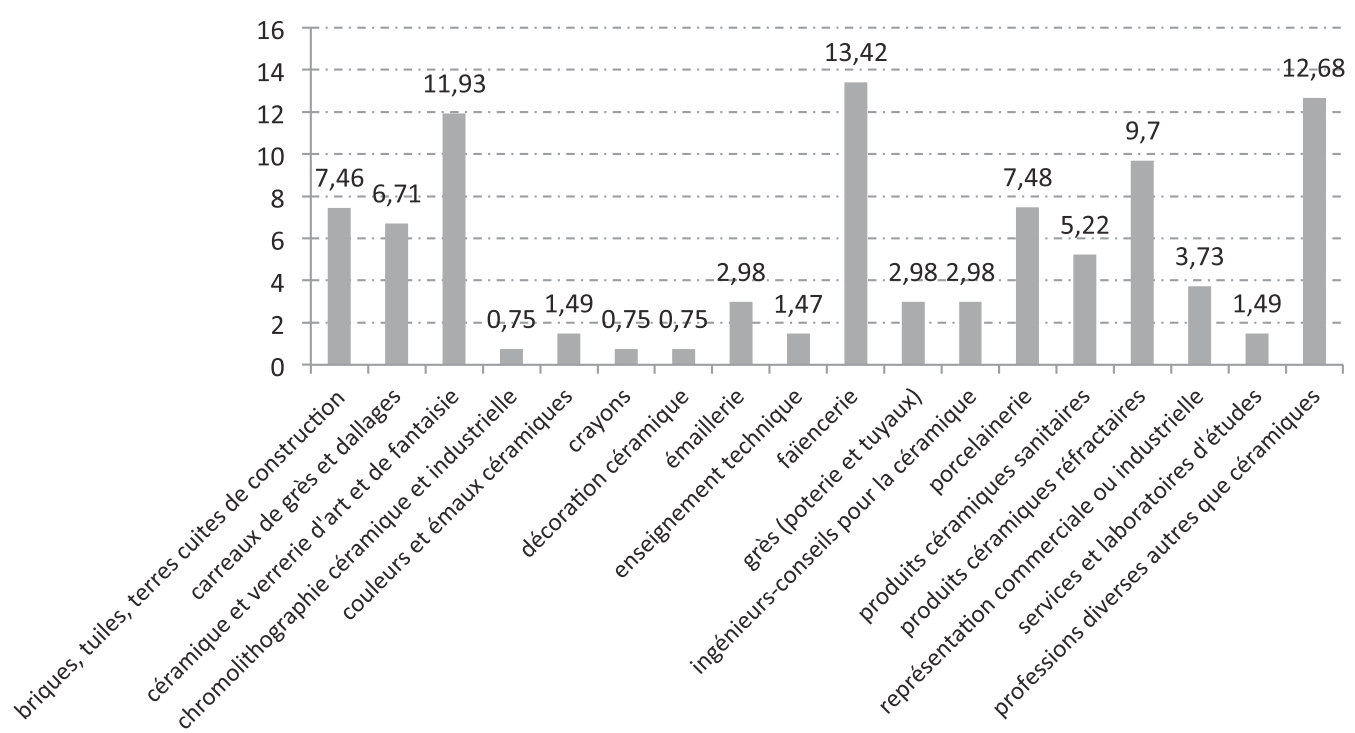

Source: Bureau universitaire de statistique et de documentation scolaires et professionnelles, Ecole nationale supérieure de céramique, $1^{\text {er }}$ juillet 1943

Figure 4: Répartition industrielle des anciens élèves, 1943, en \% (Bureau universitaire de statistique et de documentation scolaires et professionnelles, École nationale supérieure de céramique, $1^{\text {er }}$ juillet 1943). 


\section{Conclusion}

L'évolution de la formation aux métiers de la céramique est à mettre en rapport avec les transformations de la filière ellemême. Celle-ci se construit au XIX ${ }^{\mathrm{e}}$ siècle autour de la valorisation artistique de la porcelaine (Sèvres et Limoges - Haviland). Cette valorisation est à son apogée durant le dernier tiers du XIx ${ }^{\text {e }}$ siècle, alors même que les usines se mécanisent, que les ateliers laissent de moins en moins de place aux artistes et nécessitent de plus en plus d'ouvriers travaillant sur machines: la mise en place de l'enseignement technique dans les années 18701890 pâtit de ces contradictions. Autour de 1900-1910, une prise de conscience émerge tandis que la mécanisation a, dans une large mesure, joué son rôle et que les entreprises de céramique industrielle et technique se multiplient (c'est dans cette période qu'à Limoges, l'entreprise vaisselière Legrand installe un atelier pour la céramique d'équipement électrique voué à un développement considérable). Les années 19201930 marquent, pour les pouvoirs publics, le patronat et les associations professionnelles, une volonté de mise en ordre de l'enseignement technique céramique afin de prendre en compte ces évolutions industrielles.

Le tableau de la formation aux métiers de la céramique permet d'observer quatre grandes dynamiques. L'évolution du processus de production et des techniques, dans le sens de la mécanisation et d'une spécialisation des tâches, conduit à séparer exécution et création, à sortir, en quelque sorte, l'art de la fabrique. Les débouchés s'élargissent du local au national, jusqu'à l'international (en 1907, les deux tiers de la production vaisselière de Limoges sont exportés aux États-Unis). Se pose alors la question des modes de consommation et des changements de goût des consommateurs qui nécessitent d'adapter les modèles rapidement et par conséquent une prompte adaptation de la main-d'œuvre. La formation du personnel paraît d'autant plus essentielle. Celle-ci s'inscrit dans un mouvement de nationalisation de l'enseignement, confronté à la relative inertie des spécialisations productives sur une base géographique. Face à ces enjeux, les acteurs publics, patronaux, professionnels, répondent par l'élaboration de réseaux d'institutions (écoles, institut, laboratoires de recherche, syndicats patronaux, associations d'anciens élèves, etc.) dont l'action vise à embrasser l'ensemble des dynamiques à l'œuvre. Il s'agit en particulier d'établir une meilleure définition des besoins de formation en distinguant les catégories d'entreprises, de main-d'œuvre, de produits et de débouchés.

Il reste à mesurer ce qui est effectif et durable dans cette action, alors que celle-ci rencontre des problèmes de financement et que les besoins en maind'œuvre et en formation demeurent importants.

\section{Notes}

1. Florent Le Bot enseigne l'histoire contemporaine à l'université d'Évry Val d'Essonne et à l'ENS Cachan. Il est chercheur au sein de l'IDHES et de l'ANR TRAVCHER, «La production de connaissance: portrait du chercheur en travailleur salarié», 2010-2013. Il a récemment dirigé, avec Virginie Albe, Gérard Bodé, Guy Brucy et Élisabeth Chatel, 
L'ENS Cachan. Le siècle d'une grande école pour les sciences, les techniques et la société, Rennes, Presses universitaires de Rennes, 2013. Contact: flebot@ ens-cachan.fr; http://www.idhe.cnrs.fr/spip. php?article68.

2. À propos de ces jeux d'acteurs, Stéphane Lembré, L'école des producteurs. Aux origines de l'enseignement technique en France, 1800-1940, Rennes, PUR, 2013.

3. C'est notamment le cas des industries des cuirs et peaux et de la chaussure. Florent Le Bot, La fabrique réactionnaire. Antisémitisme, spoliations et corporatisme dans le cuir, 1930-1950, Paris, Presses de Sciences po, 2007.

4. Dans l'état actuel de la recherche, des données statistiques complètes sur la filière restent à élaborer.

5. Alain Pauquet, La société et les relations sociales en Berry au milieu du XIX'e siècle, Paris, L'Harmattan, 1998.

6. Jean Cartier, L'art céramique des Gréber, 18681974, Paris, Somogy, 2007.

7. Michel Pigenet, «L'ENP de Vierzon et le problème de la formation professionnelle dans une ville ouvrière, 1880-1914», Revue historique, $\mathrm{n}^{\circ} 572$, t. 282/2, septembre-décembre 1989 , p. 367-389.

8. À la suite de la crise de 1830 , des artistes parisiens s'installent à Limoges pour se consacrer au modelage et à la décoration de la porcelaine. En 1833, on y dénombre douze ateliers indépendants (environ 120 décorateurs) qui interviennent pour l'essentiel sur la production de fantaisie.

9. Clément Serre, «L'entreprise Haviland à Limoges de 1842 à 1892», mémoire de master 2, sous la dir. de Dominique Barjot, université Paris IV Sorbonne, 2010.

10. Florent Le Bot «La porcelaine de Limoges: décorée ou en blanc? (1840-1940)», dans Pierre Lamard et Nicolas Stoskopf (dir.), Art et industrie, $\mathrm{XVIII}^{e}$-XXI ${ }^{e}$ siècle, Paris, Picard, 2013, p. 165-176.

11. AD Haute-Vienne, archives de la manufacture de porcelaines Haviland, $23 \mathrm{~J}$ 7, lettre de Charles Haviland à Adrien Dubouché, 27 juin 1878.

12. AD Haute-Vienne, $23 \mathrm{~J} 69$, projets de décors de Théodore Davis pour le service «Hayes», 1880; Nora Travis, Evolution of Haviland china design, Atglen (USA), Schiffer publishing Ltd, 2000, p. 105-106.

13. Camille Grellier, L'industrie de la porcelaine en Limousin, ses origines, son évolution, son avenir, Paris, Émile Larose, 1909, p. 332.

14. Pierre Lamard, Nicolas Stoskopf, Introduction, dans P. Lamard et N. Stoskopf (dir.), Art et industrie, XVIII ${ }^{e}$-XXI $I^{e}$ siècle, op. cit., p. 10.
15. Jean-Paul Bouillon, Félix Bracquemond et les arts décoratifs. Du japonisme à l'art nouveau, Paris, Réunion des musées nationaux, 2005, p. 84-168.

16. Renaud d'Enfert, L'enseignement $d u$ dessin en France. Figure humaine et dessin géométrique, 1750-1850, Paris, Belin, 2003, notamment p. 32-36.

17. Yves Deforge, Le graphisme technique. Son histoire et son enseignement, Seyssel, Champ Vallon, 1981.

18. Bénédicte Sardin, "L'École nationale des arts décoratifs des origines à 1914: création, apogée, déclin", maîtrise d'histoire sous la dir. de Jean El Gammal, université de Limoges, 1997, p. 15-17; Chantal Meslin-Perrier, Adrien Dubouché. Un musée, un mécène, Paris, Réunion des musées nationaux, 1990.

19. Pour une analyse sémantique précise de l'usage, au XIx ${ }^{\mathrm{e}}$ siècle, des expressions "art appliqué», «art industriel», «industrie d'art» et "art décoratif», au singulier comme au pluriel, Jean-François Lumeau, "Art et industrie au XIX siècle: des arts industriels aux industries d'art», dans P. Lamard et N. Stoskopf (dir.), Art et industrie, XVIII ${ }^{e}$-XXle siècle, op. cit., p. 17-24.

20. «Distribution des prix à l'École municipale des beaux-arts appliqués à l'industrie. Discours d'Henri Ardant», Le Courrier du Centre, 11 août 1875, cité dans B. Sardin, «L'École nationale des arts décoratifs des origines à $1914 \ldots »$, op. cit., p. 18.

21. Nadège Sougy, «Du beau et de l'utile. Les qualités des fabrications industrielles, 1840-1870", dans P. Lamard et N. Stoskopf (dir.), Art et industrie, XVIII ${ }^{e}-\mathrm{XXI}{ }^{e}$ siècle, op. cit., p. 26.

22. Bernard Jacqué, "Créer des motifs pour l'impression à Mulhouse au XIX $x^{e}$ siècle: la formation et ses limites", dans P. Lamard et N. Stoskopf (dir.), Art et industrie, XVIII ${ }^{-}$-XXI ${ }^{e}$ siècle, op. cit., p. 135136.

23. Stéphane Laurent, L'art utile. Les écoles d'arts appliqués sous le Second Empire et la Troisième République, Paris, L'Harmattan, 1998, p. 127.

24. «Distribution des prix à l'École municipale des beaux-arts appliqués à l'industrie. Discours d'Adrien Dubouché », Le Courrier du Centre, 5 avril 1877, cité dans B. Sardin, «L'École nationale des arts décoratifs des origines à $1914 \ldots »$, op. cit., p. 38- 39.

25. C. Grellier, L'industrie de la porcelaine en Limousin..., op. cit., p. 378.

26. S. Laurent, L'art utile. Les écoles d'arts appliqués..., op. cit.

27. Ibid., p. 131.

28. Ibid., p. 130, note 163.

29. Ibid., p. 125. 
30. C. Grellier, L'industrie de la porcelaine en Limousin..., op. cit., p. 213.

31. Marius Vachon, Les industries d'art, les écoles et les musées d'art industriel en France, Nancy, Berger-Levrault, 1897, p. 211.

32. Fl. Le Bot «La porcelaine de Limoges: décorée ou en blanc? », op. cit.

33. Michel Pigenet, «Les ouvriers du Cher (fin $\mathrm{XVIII}^{\mathrm{e}}$ siècle-1914): travail, espace et conscience sociale", Montreuil, Institut d'histoire, 1990, préf. Georges Séguy, postf. M. Agulhon.

34. M. Pigenet, «L'ENP de Vierzon...», op. cit.

35. Ibid., p. 376.

36. Pour un point récent sur le sujet, $\mathrm{S}$. Lembré, L'école des producteurs..., op. cit.

37. Journal officiel de la République française (JORF), 9 juillet 1881.

38. Voir un ensemble de cartes postales de $l^{\prime}$ ENP de Vierzon, datant du début du $x^{\mathrm{e}}$ siècle, accessible sur le site http://pougnyjp.canalblog.com/albums/enp_vierzon/ (consulté le $1^{\text {er }}$ novembre 2013).

39. M. Pigenet, «L'ENP de Vierzon...», op. cit., p. 377.

40. Ibid., p. 378.

41. $\mathrm{AN}, \mathrm{F} / 17 / 14355$, lettre du directeur à l'inspecteur d'académie, 30 juillet 1893, cité ibid., p. 387.

42. Ibid., rapport du directeur au conseil d'administration, 18 juillet 1896, cité ibid., p. 388.

43. Jean-Pierre Desbordes, Claude Richoux, L'École nationale professionnelle Henri Brisson de Vierzon, Châteauroux, La Bouinote, 2011, p. 65-67.

44. Revue de céramique. Bulletin mensuel de la Section professionnelle de céramique de Vierzon, $\mathrm{n}^{\circ} 3$, 15 juillet 1925.

45. Florent Le Bot, «De quoi la porcelaine de Limoges est-elle le nom? L'affaire de la porcelite tchèque. Années 1930 », Entreprises et histoire, dossier dirigé par Nadège Sougy, à paraître.

46. Concernant les ordres d'enseignement, voir les travaux de Jean-Michel Chapoulie et, notamment, L'école d'État conquiert la France. Deux siècles de politique scolaire, Rennes, PUR, 2010.

47. L'ENET devient supérieure, ENSET, en 1932, Florent Le Bot, Virginie Albe, Gérard Bodé, Guy Brucy et Élisabeth Chatel (dir.), L'ENS Cachan. Le siècle d'une grande école pour les sciences, les techniques, la société, Rennes, PUR, 2013.

48. Au terme de ses statuts, les ministères de $l^{\prime}$ Instruction publique et des Beaux-Arts et du Commerce et de l'Industrie sont représentés au sein de son conseil d'administration; les autres membres sont choisis parmi des industriels, des savants et des artistes du secteur, JORF, Institut de céramique française, statuts, titre II, art. 4, 12 août
1920, p. 11710 ; Archives de la manufacture nationale de Sèvres, carton U31, dossier 19, Institut de la céramique française.

49. Union céramique et chaufournière de France, La céramique, revue mensuelle illustrée, t. XVII, 1914, p. 19.

50. Ibid., t. XX, 1917, p. 68-69.

51. Florent Le Bot, Cédric Perrin, «Mobiliser l'industrie de la chaussure, mobiliser ses territoires», Terrains \& Travaux, n 19 , 2011, p. 205-224.

52. Son premier président est Jules Loebnitz, artisan de sa mise en œuvre et ancien président de l'Union céramique et chaufournière, La céramique, t. XX, 1917, séance du comité directeur du 7 novembre 1917, p. 210.

53. JORF, Institut de céramique française, 12 août 1920, p. 11710.

54. Ibid., statuts, titre $1^{\text {er, }}$, art. $1^{\text {er }}$.

55. Ibid., statuts, titre $1^{\text {er }}$, art. $2^{\text {e }}$.

56. Ibid.

57. Association amicale des anciens élèves de l'École nationale supérieure de céramique de Sèvres, Bulletin annuel, $\mathrm{n}^{\circ}$ 20, 1933, p. 67.

58. Outre le président de l'ICF et des membres désignés parmi son conseil d'administration, ce comité se compose de sept membres de droit: le chef de la division de l'Enseignement et des manufactures nationales au ministère de l'Instruction publique et des Beaux-Arts, le directeur de $l^{\prime}$ Enseignement technique au ministère du Commerce et de l'Industrie (puis de l'Instruction publique), l'administrateur de la manufacture nationale de Sèvres, le chef du laboratoire d'essais de la manufacture, un directeur d'école professionnelle désigné par le ministère du Commerce et de l'Industrie, le censeur des études de Sèvres, JORF, Institut de céramique française, statuts, titre II, article 13.

59. Rapport et délibérations du Conseil général de l'Oise, $1^{\text {re }}$ session 1930, 6 mai 1930, p. 115-119 (sur Gallica).

60. Geo Lechevallier-Chevignard, «L'enseignement de la céramique en France», La céramique, t. XXXVII, $\mathrm{n}^{\circ}$ 555, juin 1934, p. 129.

61. Le premier président de l'Association est Léon Yeatman, La céramique, t. XXXVI, $\mathrm{n}^{\circ}$ 538, janvier 1933, p. 299.

62. Par exemple, Association de l'enseignement technique céramique, Recueil des communications $d u$ congrès technique de l'industrie céramique, Paris, Institut de céramique française, 1939.

63. Georges Rivière, "La céramique", Les arts du feu. Céramique, verrerie, vitraux, émaux, revue mensuelle, mai $1938, \mathrm{n}^{\circ} 1$, p. 2-3.

64. Voir également la communication de Geo Lechevallier-Chevignard, directeur de la manu- 
facture nationale de céramique de Sèvres lors du congrès international du verre et de la céramique, Milan, juin 1933, repris dans G. Lechevallier-Chevignard, «L'enseignement... », op. cit., p. 126-131.

65. Hippolyte Luc, «Les arts du feu. L'enseignement technique», Les Arts du feu. Céramique, Verrerie, vitraux, émaux, revue mensuelle, mai $1938, \mathrm{n}^{\circ} 1$, p. 1.
66. La céramique, 1923, p. 39, et 1933, p. 299.

67. S. Laurent, L'art utile. Les écoles d'arts appliqués..., op. cit., p. 103.

68. Ibid., p. 136-138.

69. La céramique, 1923, p. 39, et 1933, p. 299.

70. Bulletin de l'Association des anciens élèves, $1934, \mathrm{n}^{\circ} 22$, p. 32-33 